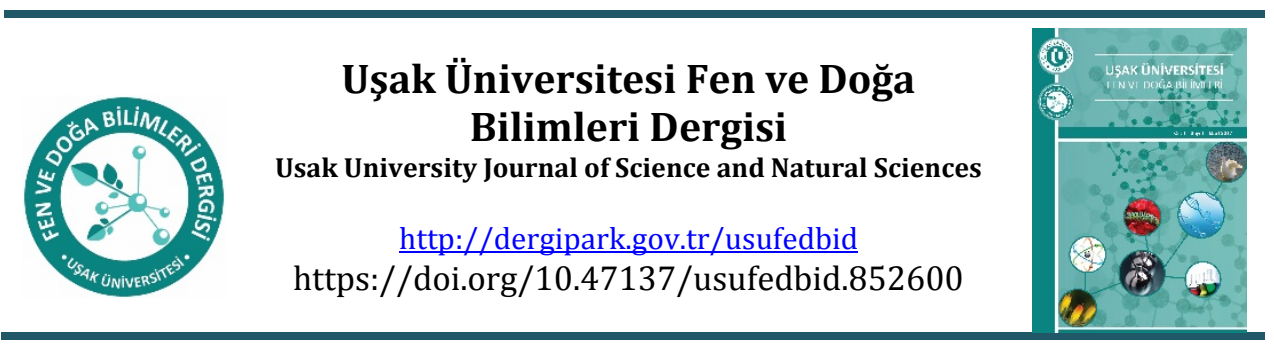

Araştırma makalesi

\title{
Prime Numbers in Layers
}

\author{
Cengiz ŞENER
}

Ministry of Education, Bafra ÜÇpınar Secondary School, Samsun, TURKEY

\begin{abstract}
In this article, unlike the known deterministic and probabilistic methods used in determining prime numbers, a new type of deterministic method based on only matrice analysis will be proven without creating a designed equation and without writing preliminary assumptions and prerequisites. For this, it will be shown that the places $30 \mathrm{k}+1,30 \mathrm{k}+7,30 \mathrm{k}+11,30 \mathrm{k}+13,30 \mathrm{k}+17,30 \mathrm{k}+19,30 \mathrm{k}+23,30 \mathrm{k}+29$ form equvailance classes of the elements of the group U(30). These eight locations will be referred to as eight layers and it will also be proven that the prime numbers are distributed equally across eight layers (equidistribution principle) in the closed system. With this new matrices method, it is also reduced the processing load considerably.
\end{abstract}

Keywords: Prime numbers in equvailance classes, regular primes, equal distribution, unconditional new deterministic matrices method.

\section{Özet}

Bu yazıda, asal sayıların belirlenmesinde kullanılan, bilinen deterministik ve olasılıkçı yöntemlerden farklı olarak, tasarlanan bir denklem oluşturmadan, ön kabuller ve ön şartlar yazmadan, sadece matris analizlerine dayanan, yeni bir tip deterministik yöntemi kanıtlamaktır. Bunun için, $30 \mathrm{k}+1$, $30 \mathrm{k}+7,30 \mathrm{k}+11,30 \mathrm{k}+13,30 \mathrm{k}+17,30 \mathrm{k}+19,30 \mathrm{k}+23,30 \mathrm{k}+29, \quad \mathrm{U}(30)$ grubunun eşitlik sinıflarından birisini oluşturduğu gösterilecektir. Bu sekiz yer, sekiz katman olarak adlandırılıp, kapalı sistem içinde asal sayıların sekiz katmana eşit olarak dağıldığı da (eşit dağılım ilkesi/ equidistribution principle ) kanıtlanacaktır. Bu yeni matrisler yöntemiyle, işlem yükünün oldukça azaltıldığı da gösterilecektir.

Anahtar Kelimeler: Eşitlik sınıflarındaki asal sayılar, düzenli asallar, eşit dağılım, şartsız yeni deterministik matrisler yöntemi.

(C)2021 Usak University all rights reserved.

\section{Introduction}

For centuries, in mathematics one of the most important problems in the field of number theory is the determination of prime numbers. For this purpose, the process that started with the Sieve of Eratosthenes (240 BC) and gained momentum with Fermat's

\footnotetext{
*Corresponding author:

E-mail: cengizsener299@gmail.com

ORCID ID: 0000-0002-5988-3472
}

(C)2021 Usak University all rights reserved. 
Little Theorem. For the prime number $\mathrm{p}$, which does not divide a number a; it is based on the satisfaction of the equality $a^{p-1}=1(\bmod \mathrm{p})$. Although this method is not exactly correct, it has formed the basis of many new deterministic algebraic methods in the future. Some of these are the AKS [1] primality test in 2004, the Miller [2] primality test in 1975 and the Rabin [3] primality test in 1980. Also in 1974, Solovay and Strassen [4] developed the primality test that could work under the extended Riemann's Hypothesis. In 1983, Adleman, Pomerance and Rumely [5] created a method based on Miller's primality test. In 1986, Goldwasser and Killian [6] found a primality method by using elliptic curves. In1992, Adleman and Huang [7] developed this method.

Besides all these deterministic tests, in 1996 Ronald Joseph Burthe, Jr. [8], in 1995 F. Arnault [9] and in 1998 J. Grantham [10] developed some of the probabilistic tests. However, with the contributions of various complexity theorems that emerged in the 1960s, primality tests had more effective and productive ways of usage. In 1967, Blum [11] and Hartley Rogers Jr. [12], and in 1972, Borodin [13] published some of the studies about complexity theories.

The purpose of this article is to introduce a new type of unconditional deterministic primality test, different from all known deterministic and probabilistic primality tests. For this purpose, in section 2, the layers system that forms the basis of the new type of primality test and the results of this system will be shown. In section 3, a new type of primilaty test will be introduced.

\section{Preliminaries: The Foundatin of Eight Layers}

The basic idea of the article starts with the knowledge that primes after 7 cannot be placed in places where multiples of the primes 2,3,5. Since the products of the primes 2,3 and 5 is 30 , other primes can be only located in the places $30 k+1,30 k+7,30 k+11$, $30 k+13,30 k+17,30 k+19,30 k+23,30 k+29$. After that, each section of $30 k$ and $30 k+29$ will be called 'package' (Б). (The section 1-30 taken as the zeroth package.) We named it this way because the matrice method is based on determining in which 30 section each prime number is. Also, there are two reasons why the eight places are called 'layers'. The first reason is; there are more than one equvailance classes of the elements of the group U(30). For example; even numbers form an equvailance class in modula 30 or also odd numbers do. Furthermore, it can be added other examples. But, in modula 30 , only one equvailance class which is formed by the place $30 \mathrm{k}+1,30 \mathrm{k}+7,30 \mathrm{k}+11,30 \mathrm{k}+13,30 \mathrm{k}+17,30 \mathrm{k}+19,30 \mathrm{k}+23,30 \mathrm{k}+29$ is significant for distribution of prime numbers. So it has to be emphasized the difference of this equvailance class from others. Then the second reason is that if it is thought that the sections of 30 's are placed side by side, the numbers in the form of $1,31,61,91, \ldots$ $(30 k+1)$ are arranged side by side. This situation horizontally forms a layer. This is class [1] which is also $K_{1} .[1]=K_{1}$. For the numbers $7,37,67,97, \ldots,(30 k+7)$ are arranged side by side. They form class [7] which is also $K_{2}$. [7]= $K_{2}$. Similar sequences occur for the other 11,13,17,19, 23 and 29 primes. Thus, the values of these eight prime numbers arranged side by side in 30 steps form their classes. So it is obtained $[11]=K_{3},[13]=K_{4},[17]=K_{5},[19]=K_{6},[23]=K_{7},[29]=K_{8}$. These classes 
compose equvailance class that is proved in Lemma 2.1. Furthermore, when the prime number is mentioned from now on, only the prime numbers, except the primes 2,3 and 5 , which are placed in layers, will be meant. Also, after that, eight layers will be used instead of eight classes, [1] $=K_{1},[7]=K_{2},[11]=K_{3},[13]=K_{4},[17]=K_{5},[19]=$ $K_{6},[23]=K_{7}, \quad[29]=K_{8}$.

Lemma 2.1. The set $S=\{1,7,11,13,17,19,23,29\}$ form a group under the operation of multiplication in mod 30 .

Proof. The Cayley table of set $\mathrm{S}$ in U(30) proves Lemma 2.1.

\begin{tabular}{c|cccccccc}
$\mathrm{U}(30)$ & 1 & 7 & 11 & 13 & 17 & 19 & 23 & 29 \\
\hline 1 & 1 & 7 & 11 & 13 & 17 & 19 & 23 & 29 \\
7 & 7 & 19 & 17 & 1 & 29 & 13 & 11 & 23 \\
11 & 11 & 17 & 1 & 23 & 7 & 29 & 13 & 19 \\
13 & 13 & 1 & 23 & 19 & 11 & 7 & 29 & 17 \\
17 & 17 & 29 & 7 & 11 & 19 & 23 & 1 & 13 \\
19 & 19 & 13 & 29 & 7 & 23 & 1 & 17 & 11 \\
23 & 23 & 11 & 13 & 29 & 1 & 17 & 19 & 7 \\
29 & 29 & 23 & 19 & 17 & 13 & 11 & 7 & 1
\end{tabular}

Furthermore, according to lemma 2.1 and its proof, corollary 2.2 and corollary 2.3 are true.

Corollary 2.2. The set S forms an equvailance class.

After that, 'pseudo prime (PP)' expression will be used for numbers that are not prime numbers in the closed system. This expression was defined by many mathematicians in the process that started with Fermat's little theorem. The reason for using the same expression, this known expression is suitable for the purpose of understanding non-prime numbers in layers.

Corollary 2.3. When one of the layer is multiplied with all layers, it is again obtained all layers one by one.

Theorem 2.4. Let $n \in N, \quad \mathrm{n} \geq 4, \quad \mathrm{p}_{\mathrm{n}}$ be the $\mathrm{nth}$ prime number and $C_{n}=2 \cdot 3 \cdot 5 \cdot 7 \cdot 11 \ldots p_{n}$

For every $p_{n}$ in the range of $C_{n}$, there are only

$P P_{n}=\left(p_{n-1}-1\right) \cdot\left(p_{n-2}-1\right) \cdot\left(p_{n-3}-1\right) \ldots(17-1) \cdot(13-1) \cdot(11-1) \cdot(7-1) \cdot 8$

number of multiples which contains itself and the primes after itself as a multiplier.

Proof. The number of total packages $\left(b_{T}\right)$ up to $C_{n}$ is

$\mathrm{b}_{T}=\frac{C_{n}}{30}=\frac{2 \cdot 3 \cdot 5 \cdot 7 \cdot 11 \ldots p_{n}}{30}=7 \cdot 11 \ldots p_{n}$ 
Then total place of layers up to $C_{n}$ is

8. $\mathrm{b}_{T}=8 \cdot 7 \cdot 11 \cdot 13.17 \ldots p_{n}$

In these layer places, the number of multiple of 7 is;

$P P_{4}=\frac{p_{n} \cdot p_{n-1} \cdot p_{n-2} \ldots 13.11 .7 .8}{7}=p_{n} \cdot p_{n-1} \cdot p_{n-2} \ldots .13 .11 .8$

The number of multiples of 11 is;

$P P_{5}=\frac{8 . \mathrm{b}_{T}-P P_{7}}{11}=\frac{p_{n} \ldots .13 \cdot 11.7 .8-\frac{p_{n} \cdot p_{n-1} \ldots 13.11 .7 .8}{7}}{11}=\frac{p_{n} \cdot p_{n-1} \cdot 13 \cdot 11 \cdot 7 \cdot 8 \cdot\left(1-\frac{1}{7}\right)}{11}=$

$p_{n} \cdot p_{n-1} \cdot p_{n-2} \ldots .13 .6 .8 P P_{5}=p_{n} \cdot p_{n-1} \cdot p_{n-2} \ldots .13 \cdot(7-1) .8$

The number of multiples of 13 is;

$P P_{6}=\frac{8.5_{T}-P P_{7}-P P_{11}}{13}$

$P P_{6}=\frac{p_{n} \cdot p_{n-1} \ldots 13.11 .7 .8-p_{n} \cdot p_{n-1} \cdot p_{n-2} \ldots 13.11 .8-p_{n} \cdot p_{n-1} \ldots 13 \cdot(7-1) \cdot 8}{13}$

$P P_{6}=p_{n} \cdot p_{n-1} \ldots 19.17 \cdot 10.6 .8=p_{n} \cdot p_{n-1} \ldots 19.17 \cdot(11-1) \cdot(7-1) .8$
$\vdots$

$P P_{n-k}=p_{n} \cdot p_{n-1} \ldots p_{n-k+1} \cdot\left(p_{n-k-1}-1\right) \ldots(17-1) \cdot(13-1) \cdot(11-1) \cdot(7-1) \cdot 8$

$P P_{n}=\left(p_{n-1}-1\right) \cdot\left(p_{n-2}-1\right) \cdot\left(p_{n-3}-1\right) \ldots(17-1) \cdot(13-1) \cdot(11-1) \cdot(7-1) \cdot 8$.

Corollary 2.5. Let $n \in N, \mathrm{n} \geq 4, \quad \mathrm{p}_{\mathrm{n}}$ be the $\mathrm{nth}$ prime number and $\mathrm{C}_{\mathrm{n}}=2$ 3.5.7.11. . . p $\mathrm{p}_{\mathrm{n}}$. For all prime numbers from 7 to $\mathrm{p}_{\mathrm{n}}$, the multiples that cannot contain primes before itself, are always placed in layers with equal distribution in the width of $\mathrm{C}_{n}$.

Proof. The first prime in the layers is $7 \quad\left(n=4\right.$ and $\mathrm{p}_{4}=7$ and $\left.\mathrm{C}_{4}=2.3 .5 .7=210\right)$, by Theorem 2.4 the number of multiples of 7 in the width of $C_{4}$ is equal to $P P_{4}=\frac{7.8}{7}=$ 8 . When 7 is multiplied with layers in an interval $[30 p-30(p+1)]$, it gives 8 results in an interval $[210 p-210(p+1)]$. According to the Cayley table of layers (proof of Lemma 2.1), these 8 results are equally distributed in 8 layers. Only in the range of (1210), there are 7 multiples of prime 7. Because the number 1 appears as an exception. But all the other 210 pieces up to infinity includes 8 multiples of prime 7 . Within 210 pieces $(210 / 30=7$ packages $)$, there are $7.8=56$ layer places are in equal distribution. When we subtract the 8 multiples of prime 7 with equal distribution, 48 places with equal distribution remains. Although these places are not in the standard layer order $(1,7,11,13,17,19,23,29)$, when a multiple of 7 is subtracted from every layer, there are 6 places remain in every layers. These 48 places are also found when Theorem 2.4 is applied for $\mathrm{n}=5$. After that, when prime 11 is multiplied by remaining 48 places in an interval $[210 p-210(p+1)]$, the results expand in an interval $[2310 p-2310(p+1)]$. So, these new 48 results is in equal distribution, in the width of all $11.210=2310$ 
pieces except the first one. Also, these multiples do not includes 7. After that, the interval of $13.2310=30030$ is reached. When multiples of 7 in every 210 pieces and multiples of 11 (not includes 7) in every pieces of 2310 are subtracted from the total layer places in the closed system, which are equally distributed, only remains places for multiples of 13 with equal distribution. That is, when equal distribution parts are removed from the total equal distribution, the number of places remains in the equal distribution. If we generalize, as a result of all primes before $p_{n}$ are placed by equal distribution, the multiples whose count is defined according to the Theorem 2.4, placed with equal distribution in the width of $\mathrm{C}_{\mathrm{n}}$. According to this, all primes and multiples from 7 to $p_{n}$ are placed with equal distribution in the width of $C_{n}$. Also, while every sequence of multiples of primes continue equal distribution separately in themselves, even if the first primes are removed from every equidistributive sequence of multiples of each prime numbers, the equal distribution property of the sequence of multiples of prime numbers continue. It means that prime numbers go to equal distribution in closed layers system because of equal distribution of pseudo-primes. Furthermore, the proof of the theorem 2.4 gives the algebraic explanation of the corollary 2.5 and corollary 2.13 will give the algebraic proof of the corollary 2.5 .

Corollary 2.6. Let $n \in N, \mathrm{n} \geq 4, \quad \mathrm{p}_{\mathrm{n}}$ be the $\mathrm{nth}$ prime number and $\mathrm{C}_{\mathrm{n}}=2 \cdot 3 \cdot 5.7 .11 \ldots \mathrm{p}_{\mathrm{n}}$. The ratio $\left(\delta_{n}^{+}\right)$of $\mathrm{p}_{\mathrm{n}}$ regular prime and its pseudo primes in the layers becomes

$$
\delta_{n}^{+}=\frac{\left(p_{n-1}-1\right) \cdot\left(p_{n-2}-1\right) \cdot\left(p_{n-3}-1\right) \ldots(13-1) \cdot(11-1) \cdot(7-1)}{p_{n} \cdot p_{n-1} \cdot p_{n-2} \ldots .13 .11 .7}
$$

Proof. Total places in the layers up to $C_{n}$ boundary is $8 . b_{s}=8.7 .11 .13 .17 \ldots p_{n}$. Generalization of $\delta^{+}$will be reached by proportioning the multiples of a prime of $p_{n}$ to the total layer places in $C_{n}$. The number of multiples of each prime is obtained from Theorem 2.4 .

For $7 ; \quad \delta_{4}^{+}=\frac{P P_{4}}{8.5_{T}}=\frac{p_{n} \cdot p_{n-1} \cdot p_{n-2} \ldots 13.11 .8}{p_{n} \cdot p_{n-1} \cdot p_{n-2} \ldots 13.11 .7 .8}=\frac{1}{7}$

For $11 ; \delta_{5}^{+}=\frac{P P_{5}}{8 . \mathrm{b}_{T}}=\frac{p_{n} \cdot p_{n-1} \cdot p_{n-2} \ldots .13 .6 .8}{p_{n} \cdot p_{n-1} \cdot p_{n-2} \ldots .13 .11 .7 .8}=\frac{6}{7.11}$

For 13; $\delta_{6}^{+}=\frac{P P_{6}}{8 . \mathrm{b}_{T}}=\frac{p_{n} \cdot p_{n-1} \cdot p_{n-2} \ldots 17.10 .6 .8}{p_{n} \cdot p_{n-1} \cdot p_{n-2} \ldots 17.13 .11 .7 .8}=\frac{6.10}{7.11 .13}$.

For 17; $\delta_{7}^{+}=\frac{P P_{7}}{8 . b_{T}}=\frac{p_{n} \cdot p_{n-1} \cdot p_{n-2} \ldots 19.12 .10 .6 .8}{p_{n} \cdot p_{n-1} \cdot p_{n-2} \ldots .19 .17 .13 .11 .7 .8}=\frac{6.10 .12}{7.11 .13 .17}$ 
For $\mathrm{p}_{\mathrm{n}} ; \delta_{n}^{+}=\frac{P P_{n}}{8 \cdot \mathrm{b}_{T}}=\frac{\left(p_{n-1}-1\right) \cdot\left(p_{n-2}-1\right) \cdot\left(p_{n-3}-1\right) \ldots .(13-1) \cdot(11-1) \cdot(7-1)}{p_{n} \cdot p_{n-1} \cdot p_{n-2} \ldots 13 \cdot 11.7}$.

Corollary 2.7.: When the sum of the proportions of the multiples of all regular primes in the layers is; $\delta^{+}=\delta_{4}^{+}+\delta_{5}^{+}+\delta_{6}^{+}+\ldots+\delta_{n}^{+}$, so $\delta^{+}$become

$$
\delta^{+}=\sum_{k=4}^{n}\left[\prod_{r=4}^{k}\left(1-\frac{1}{p_{r-1}}\right)-\prod_{r=4}^{k}\left(1-\frac{1}{p_{r}}\right)\right]
$$

Also in the term $\left(1-\frac{1}{p_{r-1}}\right)$ when $\mathrm{p}_{\mathrm{r}-1}=\mathrm{p}_{3}$ for $\mathrm{r}=4, \mathrm{p}_{3}=5$ and $\left(1-\frac{1}{p_{3}}\right)=1$ is used because the prime 5 is not in layers.

Proof. If the sum $\delta^{+}=\delta_{4}^{+}+\delta_{5}^{+}+\delta_{6}^{+}+\ldots+\delta_{n}^{+}$is written in detailed, it is obtained that

$$
\begin{aligned}
& \delta^{+}=\frac{1}{7}+\frac{(7-1)}{7 \cdot 11}+\frac{(7-1) \cdot(11-1)}{7 \cdot 11 \cdot 13}+\frac{(7-1) \cdot(11-1) \cdot(13-1)}{7 \cdot 11 \cdot 13 \cdot 17}+\ldots+\frac{\left(p_{n-1}-1\right) \cdot\left(p_{n-2}-1\right) \ldots \cdot(13-1) \cdot(11-1) \cdot(7-1)}{p_{n} \cdot p_{n-1} \ldots 13 \cdot 11 \cdot 7} \\
& \delta^{+}=\sum_{k=4}^{n}\left[\left(\frac{1}{p_{k}}\right) \cdot \prod_{r=4}^{k}\left(1-\frac{1}{p_{r-1}}\right)\right]=\sum_{k=4}^{n}\left[\left(-\left(1-\frac{1}{p_{k}}\right)+1\right) \cdot \prod_{r=4}^{k} \cdot\left(1-\frac{1}{p_{r-1}}\right)\right] \\
& \delta^{+}=\sum_{k=4}^{n}\left[\prod_{r=4}^{k}\left(-\left(1-\frac{1}{p_{r}}\right)\right)\left(1-\frac{1}{p_{r-1}}\right)+\prod_{r=4}^{k}\left(1-\frac{1}{p_{r-1}}\right)\right] \\
& \delta^{+}=\sum_{k=4}^{n}\left[\prod_{r=4}^{k}\left(1-\frac{1}{p_{r-1}}\right)-\prod_{r=4}^{k}\left(1-\frac{1}{p_{r}}\right)\right] .
\end{aligned}
$$

Corollary 2.8. Let $n \in N, \mathrm{n} \geq 4, \mathrm{p}_{\mathrm{n}}$ be the $\mathrm{nth}$ prime number and $C_{n}=2 \cdot 3 \cdot 5.7 .11 \ldots p_{n}$. Up to $C_{n}$ the sum of the ratios of the primes that couldn't get regular in layers (irregular primes) and the multiples they have created among themselves is

$$
\delta_{n}^{-}=\delta^{-}=\prod_{k=4}^{n}\left(1-\frac{1}{p_{k}}\right)
$$

Proof. In the closed layer system, it is obvious that the sum of the total ratio of multiples of regular primes and the total ratio of multiples of irregular primes becomes 1 . That is

$$
\delta^{+}+\delta^{-}=1
$$

Then $\delta^{-}=1-\delta^{+}$. Let's generalize the ratio of multiples of irregular primes by finding them separately for each term. Let $\delta_{n}^{-}$be after n-th prime starting with the $(n+1)$ th prime up to $C_{n}$ the total ratio of irregular primes and the pseudo primes formed between themselves. Then we obtain

$$
\delta_{4}^{-}=1-\delta_{4}^{+}=1-\frac{1}{7}=\frac{6}{7}
$$




$$
\begin{aligned}
& \delta_{5}^{-}=1-\delta_{4}^{+}-\delta_{5}^{+}=\frac{6}{7}-\frac{6}{7.11}=\frac{6}{7}\left(1-\frac{1}{11}\right)=\frac{6}{7} \cdot \frac{10}{11}=\left(1-\frac{1}{7}\right) \cdot\left(1-\frac{1}{11}\right) \\
& \delta_{6}^{-}=1-\delta_{4}^{+}-\delta_{5}^{+}-\delta_{6}^{+} \\
& \delta_{6}^{-}=\frac{6}{7} \cdot \frac{10}{11}-\frac{6.10}{7.11 .13}=\frac{6}{7} \cdot \frac{10}{11} \cdot\left(1-\frac{1}{13}\right)=\frac{6}{7} \cdot \frac{10}{11} \cdot \frac{12}{13}=\left(1-\frac{1}{7}\right) \cdot\left(1-\frac{1}{11}\right) \cdot\left(1-\frac{1}{13}\right)
\end{aligned}
$$$$
\delta_{n}^{-}=1-\delta_{4}^{+}-\delta_{5}^{+}-\delta_{6}^{+}-\ldots-\delta_{n}^{+}=\left(1-\frac{1}{7}\right) \cdot\left(1-\frac{1}{11}\right) \cdot\left(1-\frac{1}{13}\right) \ldots\left(1-\frac{1}{\mathrm{p}_{\mathrm{n}}}\right)
$$

Then when the limit goes to $\mathrm{Cn}=2 \cdot 3 \cdot 5.7 .11 \ldots \mathrm{p}_{\mathrm{n}}, \quad \delta_{n}{ }^{-}=\delta^{-}=\prod_{k=4}^{n}\left(1-\frac{1}{p_{k}}\right)$ is obtained.

Corollary 2.9. In the closed layers system, within the conditions of the Corollary 2.7. , Corollary 2.8. and the equation (7), the total ratio of all primes and pseudo primes is

$$
\sum_{k=4}^{n}\left[\left(\frac{1}{p_{k}}\right) \cdot \prod_{r=4}^{k}\left(1-\frac{1}{p_{r-1}}\right)\right]+\prod_{k=4}^{n}\left(1-\frac{1}{p_{k}}\right)=1
$$

The distribution of all prime numbers except the primes of 2,3,5 occurs according to the equality of (8).

Corollary 2.10. Let $n \in N, \mathrm{n} \geq 4, \quad \mathrm{p}_{\mathrm{n}}$ be the nth prime number and $\mathrm{C}_{\mathrm{n}}=2$ 3.5.7.11. . . $\mathrm{p}_{\mathrm{n}}$. According to the Corollary 2.5., all regular primes and multiples from 7 to $\mathrm{p}_{\mathrm{n}}$ are in equal distribution, when this regular region is subtracted from the total equal distribution in the closed system, the primes in the remaining irregular region and the pseudo primes they create among themselves are also placed in layers with equal distribution. In other words, like the sum of the regular region, the sum of the irregular region is placed on the layers with equal distribution.

Corollary 2.11. Let $n \in N, \mathrm{n} \geq 4, \quad \mathrm{p}_{\mathrm{n}}$ be the $\mathrm{nth}$ prime number and $\mathrm{C}_{\mathrm{n}}=2$ 3.5.7.11... $\mathrm{p}_{\mathrm{n}}$. The placement of irregular primes in the layers maintains its equal distribution character within the irregular region.

Proof. (1) Primes and multiples from 7 to $\mathrm{p}_{\mathrm{n}}$, forming the regular region, are equally distributed in $\mathrm{C}_{\mathrm{n}}$ parts according to the Corollary 2.5 .

(2) According to Corollary 2.10., like the sum of the regular region, the sum of the irregular region is placed on the layers with equal distribution.

(30 )According to Lemma 2.1. and Corollary 2.4, pseudo primes maintain their equal distribution order. 
(4) Let $\mathrm{k}$ irregular primes be from the prime $p_{n}$ to $C_{n}$. There is a $C_{n+k}$ boundary where all of these primes will become regular. As $n$ goes to infinity, for all irregular primes in the interval $\left[p_{n}, C_{n}\right]$ there exist a $C_{n+k}$ boundary such that these irregular primes must be in absolute equal distribution. The algebraic proof and result of this item is given in Corollary 2.13.

After all; according to the items (1), (2), (3) and (4), irregular primes within each $\mathrm{C}_{n}$ limit have the character of maintaining equal distribution, even if they are not in absolute equal distribution before reaching their boundaries. The main difference of equal distribution between regular and irregular regions is; in each $C_{n}$ boundary while regular primes and multiples are in absolutely equal distribution, there is no absolute equal distribution in the primes of the irregular section. While in some layer the number of primes are equal, in some other layers there may be minor differences. At each new boundary, the number of primes in any layer cannot be dominant to the number of primes in other layers, that is, the number of primes in one continuous layer is more or less than the number of primes in other layers, they maintain equal distribution by fluctuating. In the equivalence classes (layers system), since the existence of a $C_{n}$ boundary in which each prime will become regular is certain and the formation of pseudo primes maintains their equal distribution, all regular and irregular primes maintain equal distribution.

Corollary 2.12. Let $n \in N, \mathrm{n} \geq 4, \quad \mathrm{p}_{\mathrm{n}}$ be the $\mathrm{nth}$ prime number and $\mathrm{C}_{\mathrm{n}}=2$ 3.5.7.11 $\ldots \mathrm{p}_{\mathrm{n}}$. The total number of places $\left(D^{-}\right)$of the primes $\left(D_{p}{ }^{-}\right)$in irregular region and the pseudo primes $\left(D_{p p}{ }^{-}\right)$that they created among themselves in the $C_{n}$ range is $D^{-}=\left(D_{p}{ }^{-}\right)+\left(D_{p p}{ }^{-}\right)$and

$$
D^{-}=8 \cdot(7-1) \cdot(11-1) \cdot(13-1) \ldots\left(p_{n}-1\right)
$$

Proof. The result is obtained by the product of equation (3) which is the total number of places and equation (6) which is the ratio of the irregular region in the $C_{n}$ range.

Corollary 2.13. Let $\mathrm{n} \in \mathrm{N}, \mathrm{p}_{\mathrm{n}}$ be the $\mathrm{nth}$ prime number, then

$$
\lim _{n \rightarrow \infty}\left[\sum_{k=4}^{n}\left[\left(\frac{1}{p_{k}}\right) \cdot \prod_{r=4}^{k}\left(1-\frac{1}{p_{r-1}}\right)\right]\right]=1 .
$$

Proof. According to the equation (8),

$$
\sum_{k=4}^{n}\left[\left(\frac{1}{p_{k}}\right) \cdot \prod_{r=4}^{k}\left(1-\frac{1}{p_{r-1}}\right)\right]+\prod_{k=4}^{n}\left(1-\frac{1}{p_{k}}\right)=1
$$

If we take the limit of both sides, we obtain

$$
\begin{aligned}
& \lim _{n \rightarrow \infty}\left[\sum_{k=4}^{n}\left[\left(\frac{1}{p_{k}}\right) \cdot \prod_{r=4}^{k}\left(1-\frac{1}{p_{r-1}}\right)\right]+\prod_{k=4}^{n}\left(1-\frac{1}{p_{k}}\right)\right]=\lim _{n \rightarrow \infty} 1 \\
& \lim _{n \rightarrow \infty}\left[\sum_{k=4}^{n}\left[\left(\frac{1}{p_{k}}\right) \cdot \prod_{r=4}^{k}\left(1-\frac{1}{p_{r-1}}\right)\right]\right]+\lim _{n \rightarrow \infty}\left[\prod_{k=4}^{n}\left(1-\frac{1}{p_{k}}\right)\right]=1
\end{aligned}
$$


In the Euler product, let $\zeta(\mathrm{s})=\prod_{k=1}^{\infty}\left(1-\frac{1}{p_{k} s}\right)^{-1} ; \quad p_{k}$ be k-th prime. Then it is obtained

$$
\zeta(1)^{-1}=\prod_{k=1}^{\infty}\left(1-\frac{1}{p_{k}}\right)
$$

Since $\mathrm{p}_{\mathrm{k}}=\mathrm{p}_{\mathrm{n}}$, it gets;

$$
\begin{aligned}
& \prod_{k=1}^{n}\left(1-\frac{1}{p_{n}}\right)=\prod_{k=1}^{\infty}\left(1-\frac{1}{p_{k}}\right) \text { and } \\
& \prod_{k=4}^{n}\left(1-\frac{1}{p_{n}}\right)<\prod_{k=1}^{\infty}\left(1-\frac{1}{p_{n}}\right)=\zeta(1)^{-1}
\end{aligned}
$$

Since $\zeta(1)=\infty[14], \zeta(1)^{-1}=0$ Then it is obtained

$$
\lim _{n \rightarrow \infty}\left[\prod_{k=4}^{n}\left(1-\frac{1}{p_{k}}\right)\right] \leq \lim _{n \rightarrow \infty}\left[\zeta(1)^{-1}\right]=0
$$

Since all terms of $\prod_{k=4}^{n}\left(1-\frac{1}{p_{k}}\right)$ are non-negative,

$$
\lim _{n \rightarrow \infty}\left[\prod_{k=4}^{n}\left(1-\frac{1}{p_{k}}\right)\right]=0
$$

Then it is obtained,

$$
\begin{gathered}
\lim _{n \rightarrow \infty}\left[\sum_{k=4}^{n}\left[\left(\frac{1}{p_{k}}\right) \cdot \prod_{r=4}^{k}\left(1-\frac{1}{p_{r-1}}\right)\right]\right]+\lim _{n \rightarrow \infty}\left[\prod_{k=4}^{n}\left(1-\frac{1}{p_{k}}\right)\right]=1 \\
\lim _{n \rightarrow \infty}\left[\sum_{k=4}^{n}\left[\left(\frac{1}{p_{k}}\right) \cdot \prod_{r=4}^{k}\left(1-\frac{1}{p_{r-1}}\right)\right]\right]+0=1 \\
\lim _{n \rightarrow \infty}\left[\sum_{k=4}^{n}\left[\left(\frac{1}{p_{k}}\right) \cdot \prod_{r=4}^{k}\left(1-\frac{1}{p_{r-1}}\right)\right]\right]=1
\end{gathered}
$$

Corollary 2.13 is the algebraic proof of the equiditribution of pseudo-primes which are in regular region as mentioned in corollary 2.5 and pseudo-primes in equivalence classes as mentioned in forth item of proof of corollary 2.11 .

Corollary 2.14. Let $n \in N, A_{n}$ be the $n$th prime number, then

$\sum_{k=4}^{n}\left[\left(\frac{1}{p_{k}}\right) \cdot \prod_{r=4}^{k}\left(1-\frac{1}{p_{r-1}}\right)\right]=\sum_{k=1}^{n}\left[\left(\frac{1}{p_{k}}\right) \cdot \prod_{r=1}^{k}\left(1-\frac{1}{p_{r-1}}\right)\right]=1$

Proof. According to the Corollary 2.7 and Corollary 2.13, it is obtained

$\sum_{k=4}^{n}\left[\left(\frac{1}{p_{k}}\right) \cdot \prod_{r=4}^{k}\left(1-\frac{1}{p_{r-1}}\right)\right]=\frac{1}{7}+\frac{6}{7 \cdot 11}+\frac{6 \cdot 10}{7 \cdot 11 \cdot 13}+\frac{6 \cdot 10 \cdot 12}{7 \cdot 11 \cdot 13 \cdot 17}+\ldots=1$

Then if the right part of the equation (12) written explicitly, 


$$
\begin{aligned}
& \sum_{k=1}^{n}\left[\left(\frac{1}{p_{k}}\right) \cdot \prod_{r=1}^{k}\left(1-\frac{1}{p_{r-1}}\right)\right]=\frac{1}{2}+\frac{1}{2.3}+\frac{2}{2.3 .5}+\frac{2.4}{2 \cdot 3.5 .7}+\frac{2.4 .6}{2.3 .5 .7 .11}+ \\
& \frac{2 \cdot 4 \cdot 6 \cdot 10}{2 \cdot 3 \cdot 5 \cdot 7 \cdot 11 \cdot 13}+\frac{2 \cdot 4 \cdot 6 \cdot 10 \cdot 12}{2 \cdot 3 \cdot 5 \cdot 7 \cdot 11 \cdot 13 \cdot 17}+\ldots \\
& =\frac{1}{2}+\frac{1}{6}+\frac{1}{15}+\frac{4}{15 \cdot 7}+\frac{4 \cdot 6}{15 \cdot 7 \cdot 11}+\frac{4 \cdot 6 \cdot 10}{15 \cdot 7 \cdot 11 \cdot 13}+\frac{4 \cdot 6 \cdot 10 \cdot 12}{15 \cdot 7 \cdot 11 \cdot 13 \cdot 17}+\ldots \\
& =\frac{1}{2}+\frac{1}{6}+\frac{1}{15}+\frac{4}{15} \cdot\left(\frac{1}{7}+\frac{6}{7 \cdot 11}+\frac{6 \cdot 10}{7 \cdot 11 \cdot 13}+\ldots\right)
\end{aligned}
$$

In paranthesis;

$\sum_{k=4}^{n}\left[\left(\frac{1}{p_{k}}\right) \cdot \prod_{r=4}^{k}\left(1-\frac{1}{p_{r-1}}\right)\right]=\frac{1}{7}+\frac{6}{7.11}+\frac{6.10}{7.11 .13}+\frac{6 \cdot 10.12}{7 \cdot 11.13 .17}+\ldots=1$

So, it is obtained;

$$
\begin{aligned}
& \sum_{k=1}^{n}\left[\left(\frac{1}{p_{k}}\right) \cdot \prod_{r=1}^{k}\left(1-\frac{1}{p_{r-1}}\right)\right]=\frac{1}{2}+\frac{1}{6}+\frac{1}{15}+\frac{4}{15} \cdot(1) \\
& \sum_{k=1}^{n}\left[\left(\frac{1}{p_{k}}\right) \cdot \prod_{r=1}^{k}\left(1-\frac{1}{p_{r-1}}\right)\right]=\frac{1}{2}+\frac{1}{6}+\frac{5}{15}==\frac{1}{2}+\frac{1}{6}+\frac{1}{3}=1
\end{aligned}
$$

\section{The Method of Package Numbers Matrixes}

\subsection{Preliminaries: The Linear Algebra of Pseudo-Prime Numbers}

Lemma 3.1.1. Let $\left(b_{1}, b_{2}, b_{3}\right) \in N, x, y, s=1,2,3,4,5,6,7,8 . K_{x}, K_{y}, K_{s}$ be layers and $B$ is number checked. In the product; $K_{s}$ is result layer and layer of checked number $B$. Also, $b_{\text {s }}$ is result package number and package number of checked number $\mathrm{B}$.

$\left(30 b_{1}+K_{x}\right)\left(30 b_{2}+K_{y}\right)=\left(30 b_{s}+K_{s}\right)=B$

The values of $b_{s}$ form a matrix which has all rows and columns are arithmetic sequences.

Proof: In product (1), it is obtained;

$$
\left(30 b_{1}+K_{x}\right) \cdot\left(30 b_{2}+K_{y}\right)=30 b_{1} \cdot 30 b_{2}+30 b_{1} \cdot K_{y}+30 b_{2} \cdot K_{x}+K_{x} \cdot K_{y}
$$

According to Lemma 2.1, $\mathrm{K}_{\mathrm{x}} \cdot \mathrm{K}_{\mathrm{y}}=30 \mathrm{~b}^{\prime}+\mathrm{K}_{\mathrm{s}}$.

So the equation (14) turns into;

$$
\left(30 b_{1}+K_{x}\right) \cdot\left(30 b_{2}+K_{y}\right)=30 b_{1} \cdot 30 b_{2}+30 b_{1} \cdot K_{y}+30 b_{2} \cdot K_{x}+30 b^{\prime}+K_{s}
$$

the first four terms on the right are taken into 30 parenthesis and it is obtained; 
$\left(30 b_{1}+K_{x}\right) \cdot\left(30 b_{2}+K_{y}\right)=30\left(30 b_{1} \cdot b_{2}+b_{1} \cdot K_{y}+b_{2} \cdot K_{x}+b^{\prime}\right)+K_{s}$
So; $\quad b_{s}=30 b_{1} \cdot b_{2}+b_{1} \cdot K_{y}+b_{2} \cdot K_{x}+b^{\prime}$

It is assumed that $b_{1}$ values are rows and $b_{2}$ values are columns.

For rows $\left(\mathrm{r}_{\mathrm{i}}\right)$;

$\left(r_{1}=1, b_{1}=0\right),\left(r_{2}=2, b_{2}=1\right),\left(r_{3}=3, b_{3}=2\right), \ldots,\left(r_{i}=i, b_{x}=(i-1)\right), \ldots$

For columns $\left(c_{j}\right)$;

$\left(c_{1}=1, b_{1}=0\right),\left(c_{2}=2, b_{2}=1\right),\left(c_{3}=3, b_{3}=2\right), \ldots,\left(c_{j}=j, b_{y}=(j-1)\right), \ldots$

It will be composed matrixes using either $b_{1}$ values or $b_{2}$ values in equation (18). Also, both can be used at the same time.

In addition, when used $b_{1}, b^{\prime}, K_{x}$ and $K_{y}$ are constants, value of each row changes according to $b_{2}$ as follows;

Subracting consecutive elements of a row, it is obtained;

$$
\begin{aligned}
& {\left[30 \mathrm{~b}_{1} \cdot\left(\mathrm{b}_{2}+1\right)+\mathrm{b}_{1} \cdot K_{y}+\left(\mathrm{b}_{2}+1\right) \cdot K_{x}+\mathrm{b}^{\prime}\right]-\left[30 \mathrm{~b}_{1} \cdot \mathrm{b}_{2}+\mathrm{b}_{1} \cdot K_{y}+\mathrm{b}_{2} \cdot K_{x}+\right.} \\
& \left.\mathrm{b}^{\prime}\right] \\
& \quad=30 \mathrm{~b}_{1} \cdot \mathrm{b}_{2}+30 \mathrm{~b}_{1}+\mathrm{b}_{1} \cdot K_{y}+\mathrm{b}_{2} \cdot K_{x}+K_{x}+\mathrm{b}^{\prime}-30 \mathrm{~b}_{1} \cdot \mathrm{b}_{2}-\mathrm{b}_{1} \cdot K_{y}-\mathrm{b}_{2} \cdot K_{x}-\mathrm{b}^{\prime} \\
& \quad=30 \mathrm{~b}_{1}+K_{x}
\end{aligned}
$$

Or, when used $b_{2}, b^{\prime}, K_{x}$ and $K_{y}$ are constants, value of each column changes according to $b_{1}$ like obtaining equation (21), it is accessed that;

$\left[30 b_{2} \cdot\left(b_{1}+1\right)+b_{2} \cdot K_{x}+\left(b_{1}+1\right) \cdot K_{y}+b^{\prime}\right]-\left[30 b_{1} \cdot b_{2}+b_{1} \cdot K_{y}+b_{2} \cdot K_{x}+b^{\prime}\right]$

$=30 \mathrm{~b}_{2}+K_{y}$

Consequently, according to equations (21) and (22), all rows and columns of the matrixes are arithmetic sequence. That is, all rows and columns of the matrixes have linear relations within themselves.

\section{The Result Matrix (23)}

According to equalities (18), (19), (20), (21), (22) and equation $K_{x} \cdot K_{y}=30 b^{\prime}+K_{s}$; $\mathrm{b}_{1}$ 's are rows and $\mathrm{b}_{2}$ 's are columns.

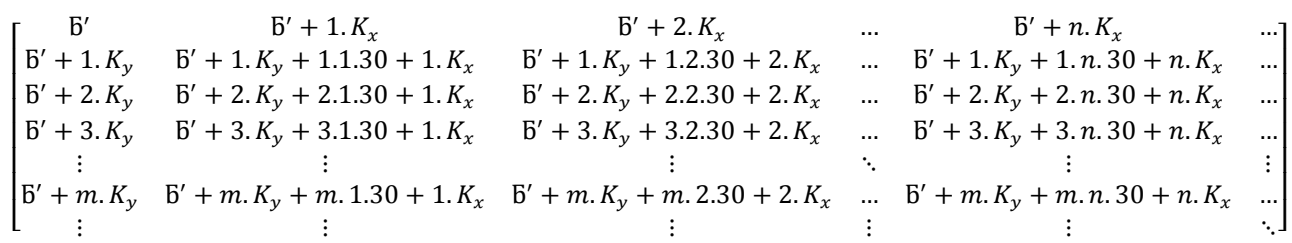


Corollary 3.1.2. According to the matrix (23) and equalities (21) and (22);

$$
\begin{aligned}
& \left(\mathrm{b}_{s}\right)_{i x j}=\mathrm{b}^{\prime}+m \cdot K_{y}+m \cdot n \cdot 30+n \cdot K_{x} \\
& \text { so } \quad \mathrm{m}=(\mathrm{i}-1)=\mathrm{b}_{1} \quad \text { and } \mathrm{n}=(\mathrm{j}-1)=\mathrm{b}_{2}
\end{aligned}
$$

Corollary 3.1.3. There are 36 multiplication possibilities of equation (15); $\mathrm{K}_{\mathrm{x}} \cdot \mathrm{K}_{\mathrm{y}}=30 \mathrm{~b}^{\prime}+\mathrm{K}_{\mathrm{s}}$.

Proof:

Table 1. Multiplication Possibilities of Equation $K_{x} \cdot K_{y}=30 b^{\prime}+K_{s}$

\begin{tabular}{|c|c|}
\hline $\mathrm{K}_{\mathrm{s}}$ & $\mathrm{K}_{\mathrm{x}} \mathrm{K}_{\mathrm{y}}$ \\
\hline 1 & $(1.1),(7.13),(11.11),(17.23),(19.19),(29.29)$ \\
\hline 7 & $(1.7),(11.17),(13.19),(23.29)$ \\
\hline 11 & (1.11), (7.23), (13.17), (19.29) \\
\hline 13 & (1.13), (7.19), (11.23), (17.29) \\
\hline 17 & (1.17), (7.11), (19.23), (13.29) \\
\hline 19 & (1.19), (7.7), (11.29), (13.13), (17.17), (23.23) \\
\hline 23 & (1.23), (11.13), (17.19), (7.29) \\
\hline 29 & (1.29), (7.17), (13.23), (11.19) \\
\hline
\end{tabular}

In 8 layers, for the $K_{s}$ layers 7, 11, 13, 17, 23 and 29, there are 4 different multiplication possibilities giving as a result. There are 6 different multiplication possibilities, giving 1 and 19 layers as a result. Consequently, there are, totally, 36 multiplication possibilities of equation (15); $K_{x} \cdot K_{y}=30 b^{\prime}+K_{s}$.

Corollary 3.1.4. There are 36 result matrix possibilities according to corollary 3.1 .3 and its proof.

Corollary 3.1.5. When it is taken $K_{x}=1$ and $b_{1}=0$ in result matrices of multiplication possibilities of $(1,7),(1,11),(1,13),(1,17),(1,19),(1,23),(1,29)$, some elements of the first rows of these seven results matrices are package numbers of the pseudo-prime numbers and some are package numbers of the prime numbers. Also, when it is taken $\mathrm{K}_{\mathrm{x}}=1, \mathrm{~K}_{\mathrm{y}}=1, \mathrm{~b}_{1}=0$ and $\mathrm{b}_{2}=0$ in result matrice of multiplication possibility of $(1,1)$, some elements of the first row and the first column of this result matrice are package numbers of the pseudo-prime numbers and some are package numbers of the prime numbers.

Proof: $\mathrm{K}_{\mathrm{x}}=1$ and $\mathrm{b}_{1}=0$, so $30 \mathrm{~b}_{1}+K_{x}=1$. The values of $\mathrm{b}_{2}$ as integers are multiplied by 1 . So these multiplications do not turn the package numbers of prime numbers to the package numbers of pseudo-prime numbers. The first rows of these seven results matrices are composed $0,1,2,3,4, \ldots$ as integers. In conclusion, some elements of the first rows of these seven results matrices are package numbers of the pseudo-prime numbers and some are package numbers of the prime numbers. In addition, the first row and the first column of result matrice of 
multiplication possibility of $(1,1)$ is in same situation. These rows or/and columns are exeptional first rows and columns.

Corollary 3.1.6. Exept for the elements of the exeptional rows and columns in corollary 3.1.5, all elements of 36 matrix possibilities of the result matrix(23) are package numbers of the pseudo-prime numbers according to corollaries 3.1.2, 3.1.3 and 3.1.4.

Theorem 3.1.7. $b^{\prime} \in N, K_{s}$ is the layer and $B=30 b^{\prime}+K_{s}$. According to corollary 3.1.5 and corollary 3.1.6, the number $\mathrm{B}$ is a prime number if the value $b^{\prime}$ is not an element of one of the resulting matrices that yields $K_{s}$ without using exeptional rows or/and columns.

Theorem 3.1.8. The determinant of a square matrix formed by four elements with locations $((a+1),(b+1)),((a+1),(d+1)),((c+1),(b+1))$ and $((c+1),(d+1))$ in any 36 result matrices is:

$\operatorname{det}\left[\begin{array}{ll}\mathrm{b}^{\prime}+a \cdot K_{y}+a \cdot b \cdot 30+b \cdot K_{x} & \mathrm{~b}^{\prime}+a \cdot K_{y}+a \cdot d \cdot 30+d \cdot K_{x} \\ \mathrm{~b}^{\prime}+c \cdot K_{y}+c \cdot b \cdot 30+b \cdot K_{x} & \mathrm{~b}^{\prime}+c \cdot K_{y}+c \cdot d \cdot 30+d \cdot K_{x}\end{array}\right]=-\mathrm{K}_{\mathrm{s}} \cdot(\mathrm{c}-\mathrm{a}) \cdot(\mathrm{d}-\mathrm{b})$.

Proof: According to equalities (19) and (20), For rows $\left(r_{i}\right) ; \quad r_{1}=(a+1), \quad p_{1}=a$ and $\mathrm{r}_{2}=(\mathrm{c}+1), \mathrm{p}_{2}=\mathrm{c}$

For columns $\left(c_{j}\right) ; \quad c_{1}=(b+1), p_{3}=b$ and $c_{2}=(d+1), p_{4}=d$. When $a, b, c, d$ values are substituted in a $2 \times 2$ square marix according to equation (18), it is formed;

$\left[\begin{array}{ll}\mathrm{b}^{\prime}+a \cdot K_{y}+a \cdot b \cdot 30+b \cdot K_{x} & \mathrm{~b}^{\prime}+a \cdot K_{y}+a \cdot d \cdot 30+d \cdot K_{x} \\ \mathrm{~b}^{\prime}+c \cdot K_{y}+c \cdot b \cdot 30+b \cdot K_{x} & \mathrm{~b}^{\prime}+c \cdot K_{y}+c \cdot d \cdot 30+d \cdot K_{x}\end{array}\right]$

When it is calculated the determinant of the matrix (26);

$$
\begin{aligned}
\operatorname{det}[(26)] & =\left(\mathrm{b}^{\prime}+a \cdot K_{y}+a \cdot b \cdot 30+b \cdot K_{x}\right) \cdot\left(\mathrm{b}^{\prime}+c \cdot K_{y}+c \cdot d \cdot 30+d \cdot K_{x}\right) \\
& -\left(\mathrm{b}^{\prime}+a \cdot K_{y}+a \cdot d \cdot 30+d \cdot K_{x}\right) \cdot\left(\mathrm{b}^{\prime}+c \cdot K_{y}+c \cdot b \cdot 30+b \cdot K_{x}\right)
\end{aligned}
$$

$\operatorname{det}[(26)]=b^{\prime} \cdot b^{\prime}+b^{\prime} \cdot c \cdot K_{y}+b^{\prime} \cdot c \cdot d \cdot 30+b^{\prime} \cdot d \cdot K_{x}+a \cdot K_{y} \cdot b^{\prime}+a \cdot K_{y} \cdot c \cdot K_{y}$ $+a \cdot K_{y} \cdot c \cdot d \cdot 30+a \cdot K_{y} \cdot d \cdot K_{x}+$ a.b.30. $\mathrm{b}^{\prime}+$ a.b.30 . c. $K_{y}+$ a.b.30 . c.d.30

+ a.b.30.d. $K_{x}+b \cdot K_{x} \cdot \mathrm{b}^{\prime}+b \cdot K_{x} \cdot c \cdot K_{y}+b \cdot K_{x} \cdot c \cdot d \cdot 30+b \cdot K_{x} \cdot d \cdot K_{x}-\mathrm{b}^{\prime} \cdot \mathrm{b}^{\prime}-\mathrm{b}^{\prime} \cdot c \cdot K_{y}$

- $b^{\prime} \cdot c \cdot b .30-\mathrm{b}^{\prime} . b . K_{x}-a \cdot K_{y} \cdot \mathrm{b}^{\prime}-a \cdot K_{y} \cdot c \cdot K_{y}-a \cdot K_{y} \cdot c \cdot b \cdot 30-a \cdot K_{y} \cdot b \cdot K_{x}$

- a.d.30. $\mathrm{b}^{\prime}$ - a.d.30.c. $K_{y}$ - a.d.30.c.b.30 - a.d.30.b. $K_{x}-d . K_{x} . \mathrm{b}^{\prime}-$ d. $K_{x} . c . K_{y}$

- d. $K_{x} . c . b .30-d . K_{x} . b . K_{x}$

$\operatorname{det}[(26)]=b^{\prime} \cdot c \cdot d \cdot 30+a \cdot K_{y} \cdot d \cdot K_{x}+a \cdot b \cdot 30 \cdot b^{\prime}+b \cdot K_{x} \cdot c \cdot K_{y}-b^{\prime} \cdot c \cdot b \cdot 30-a \cdot K_{y} \cdot b \cdot K_{x}$ - a.d.30. $\mathrm{b}^{\prime}-$ d. $K_{x}$.c. $K_{y}$ 
$\operatorname{det}[(26)]=30 . b^{\prime} \cdot($ c.d $+a \cdot b-c b-a \cdot d)-K_{x} \cdot K_{y} \cdot(-$ a.d - b.c + a.b + c.d $)$ $\operatorname{det}[(26)]=\left(30 \cdot b^{\prime}-K_{x} \cdot K_{y}\right) \cdot(c \cdot d+a \cdot b-c b-a \cdot d)$

According to equation (15) $\mathrm{K}_{\mathrm{x}} \cdot \mathrm{K}_{\mathrm{y}}=30 \mathrm{~b}^{\prime}+\mathrm{K}_{\mathrm{s}}$, so; $\quad-\mathrm{K}_{\mathrm{s}}=30 \mathrm{~b}^{\prime}-\mathrm{K}_{\mathrm{x}} \cdot \mathrm{K}_{\mathrm{y}}$

$\operatorname{det}[(26)]=-K_{s} \cdot(c \cdot d+a \cdot b-c b-a \cdot d)=-K_{s} \cdot[c \cdot(d-b)-a \cdot(d-b)]$

$\operatorname{det}[(26)]=-K_{s} \cdot(c-a) \cdot(d-b)$

\subsection{The Method of Finding Prime Numbers by Using Matrixes of Package Numbers of Pseudo- Prime Numbers:}

Definition 3.2.1. The method of finding prime numbers by using matrices of package numbers of pseudo-prime numbers will be defined step by step:

Step 1: Using equation (13), it is reached $b_{s}, K_{s}$ values.

Step 2: In equation; $\mathrm{K}_{\mathrm{x}} \cdot \mathrm{K}_{\mathrm{y}}=30 \mathrm{~b}^{\prime}+\mathrm{K}_{\mathrm{s}}$ and according to the table 1 , it is obtained $\mathrm{K}_{\mathrm{x}}, \mathrm{K}_{\mathrm{y}}$ and $\mathrm{b}^{\prime}$ values.

Step 3: Substituting the values obtained from step 1 and 2 to the matrix (23). So, it is composed all matrice possibilites ( 4 or 6 , according to table 1 ) of $\mathrm{K}_{\mathrm{s}}$.

Step 4: It is checked whether $b_{s}$ value is an element of one of the possible matrices exept for the elements of the exeptional first rows or/and columns in corollary 3.1.5.

Step 5: If it is not an element of any possible matrices, B is a prime number (Theorem 3.1.7).

Step 6: If, at least, it is one of an element of any possible result matrices (without exeptional rows or/and columns), B is a pseudo- prime number.

Step 7: For $b_{s}$ values which is found in all possible matrixes, it is obtained all multipliers of number B according to equalities (24) and equation (13).

$$
\left(30(i-1)+K_{x}\right) \cdot\left(30(j-1)+K_{y}\right)=\left(30 b_{1}+K_{x}\right)\left(30 b_{2}+K_{y}\right)=\left(30 b_{s}+K_{s}\right)=B
$$

\section{Examples 3.2.2. :}

1) $B=553243$, is it a prime number?

$553243=30.18441+13$, so $b_{s}=18441$ and $K_{s}=13$.

It has 4 matrice possibilities which are (1.13), (7.19), (11.23), (17.29).

Only the element of matrice possibility of $(17.29=30.16+13)$ gives $b_{s}=18441$ value. Also, $\mathrm{b}^{\prime}=16$. 
Using matrice (23), with respect to equation $\left(30 b_{1}+17\right)\left(30 b_{2}+29\right)=\left(30 b_{s}+\right.$ $13)=B$, the equality (18) which gives $\left(b_{s}=30 b_{1} \cdot b_{2}+b_{1} \cdot 29+b_{2} \cdot 17+\right.$ 16 ) generates the matrix where $b_{1}$ 's are rows and $b_{2}$ 's are columns.

$$
\left[\begin{array}{ccccc}
16 & 33 & 50 & 67 & \ldots \\
45 & 92 & 139 & 186 & \ldots \\
74 & 151 & 228 & 305 & \ldots \\
\vdots & \vdots & \vdots & \vdots & \ldots \\
(9064)_{313,1} & (18441)_{313,2} & (27818)_{313,3} & (37195)_{313,4} & \ldots \\
\vdots & \vdots & \vdots & \vdots & \ddots
\end{array}\right]
$$

$\mathrm{i}=313, \mathrm{j}=2$, so, according to equation (26);

$\left(30(\mathrm{i}-1)+\mathrm{K}_{\mathrm{x}}\right) \cdot\left(30(\mathrm{j}-1)+\mathrm{K}_{\mathrm{y}}\right)=(30.312+17)(30.1+29)=9377.59=$ $553243=\mathrm{B}$

Proof of example 1: The number 18441 is in the second column. According to equation (22), the difference between consecutive elements of second column is; $30 \cdot(2-1)+29=59$. It must be $33+59 \cdot \mathrm{m}=18441$ and $\mathrm{m}$ must be an integer, too.

$\mathrm{m}=\frac{18441-33}{59}=312 ; \mathrm{m}$ is an integer. $\mathrm{m}=(\mathrm{i}-1)=312$ and $\mathrm{i}=313$. So, 553243 is not a prime number.

2) $\mathrm{B}=105817$, is it a prime number?

$105817=30.3527+7$, so $b_{s}=3527$ and $K_{s}=7$.

It has 4 matrice possibilities which come from (1.7), (11.17), (13.19), (23.29). When these possible result matrices are composed according to equality (18) and matrice (23), any of 4 matrices do not include 3527 as an element. Consequently, 105817 is a prime number according to the Theorem 3.1.7.

\subsection{General Using of the Matrice Method}

According to matrice algorithm which is matrice (23), computers compose 36 matrices. Package numbers are asked to computers one by one as integers goes to infinity. It is determined in which matrices an searched integer is found or not. Furthermore, the first rows or/and columns of matrices which are composed by 1 are not included searching. There are pseudo primes in the result layers $\left(K_{s}\right)$ defined by the matrices containing the searched integer and there are primes in the result layers $\left(K_{s}\right)$ defined by the matrices which do not contain the searched integer. As a result, what needs to be done is to search an integer in which 36 matrices have as elements or not. That is, one number is asked and eight answers are received. It is not necessary to repeatedly compose 36 matrices to search prime numbers. Matrices are formed and they are used to check prime numbers infinitely. Also, for very large elements of matrices, some arrangements can be made within the matrices using linear algebra. Besides all of these, the most important 
result of this matrice method is that for finding a prime number, it does not have to been searched prime numbers. Thus, it is reduced the processing load considerably.

\section{Conclusion}

In this study, it was proved that all prime numbers except the first three prime numbers form equivalence class and that the placement of prime numbers in this system is realized with an equal distribution. In addition, it has been proved algebraically that the non-prime numbers formed by the prime numbers in this system are equally distributed. Furthermore, the new type of prime number determination method obtained depending on the advantage provided by the layer system and the linear algebra also has a much more development potential.

\section{Acknowledgement}

I thank referees for their valuable comments.

\section{References}

1. Agrawal M, Kayal N, Saxena N. Primes is in P. Ann. Math., 2004;160:781-793.

2. Miller GL. Riemann's hypothesis and tests for primality. J. Comput. Sys. Sci., 1976;13:300-317.

3. Rabin M O. Probabilistic algoritm for testing primality. J. Number Theory, 1980;12:128-138.

4. Solovay R, Strassen V. A fast Monte-Carlo test for primality. SIAM Journal on Computing, 1977;6:84-86.

5. Adleman L M, Pomerance C, Rumely R S. On distinguishing prime numbers from composite numbers. Ann. of Math., 1983;117:173-206.

6. Goldwasser S, Kilian J. Almost all primes can be quickly certified. Proc. 18th STOC., 1986;316-329.

7. Adleman L M, Huang M D. Primality testing and two dimensional Abelian varieties over finite fields. Lecture Notes in Mathematics, 1992;1512.

8. Burthe R J Jr. Further investigations with the strong probable prime test. Mathematics of Computation, 1996;65(213):373-381.

9. Arnault F. Rabin-Miller primality test: Composite numbers which pass it. Mathematics of Computation, 1995;64(209):355-361.

10. Gratham J. A probable prime test with high confidence. J. Number Theory, 1998; 72: $32-47$

11. Blum M. A machine-independent theory of the complexity of recursive functions. J. Assoc. Comput. Mach., 1967;14: 332-336.

12. Rogers H Jr. Theory of recursive functions and effective computability. New York: McGraw-Hill; 1967; MR37\#61.

13. Borodin A. Computational complexity and the existence of complexity gaps. J. Assoc. Comput. Mach., 1972;19:158-174.

14. The values of $\zeta(n)$ for small positive integer values of $n$, equation 70 . URL: https://mathworld.wolfram.com/RiemannZetaFunction.html [Document on the Internet], 2020 [cited 2020 September 10]. 